\title{
Prevenção e promoção de saúde no CAPS AD através de oficinas de psicoeducação
}

Cleonilde de Fátima Guariento', Samantha Torres², Daniel Dall'Igna Ecker³

I Psicóloga e Assistente Social, RS. Brasil.

E-mail: cleofgfaculdadepsico@gmail.com

2 Mestra em Psicologia Social e Institucional. Membra do colegiado gestor do CRR-Rede Multicêntrica/UFRGS.

Participante do grupo "Ideologia, Comunicação e Representações Sociais", coordenado pelo Dr. Pedrinho Guareschi (UFRGS).

E-mail: samantha.torres@gmail.com

${ }^{3}$ Doutorando na Universidade Federal do Rio Grande do Sul (UFRGS). Mestre em Psicologia Social e Institucional (UFRGS).

Psicólogo (PUCRS), RS, Brasil.

E-mail: daniel.ecker@hotmail.com

Recebido em: 13 jan. 2019. Aceito em: 03 mar. 2019. DOI: http://dx.doi.org/I0.21674/2448-0479.52.191-197

\section{Resumo}

Este artigo é um breve relato de experiência de um projeto de intervenção em Psicologia, com o foco na Prevenção e Promoção de Saúde, realizado em um Centro de Atenção Psicossocial Álcool e outras Drogas (CAPS AD). Através da ferramenta terapêutica de psicoeducação foram realizados oito encontros, em formato de oficina, no período de agosto a setembro de 2017, com carga horária de duas horas semanais. Participaram dos encontros 10 usuários(as) do serviço, sendo a maioria homens na faixa etária de 36 a 57 anos. As oficinas tiveram como principal dispositivo ensinar técnicas de relaxamento, através do exercício da respiração diafragmática, relacionadas a técnicas psicológicas para os sujeitos refletirem sobre si no mundo. Como resultado, os participantes relataram diminuição da ansiedade e tensão produzindo, como efeito, mudanças em seus hábitos diários, tanto no sono, como na alimentação e em relação a conflitos familiares, passando a ter uma atitude mais ativa no próprio tratamento e tirando o foco do atendimento médico e da busca pela medicalização dos sintomas. Conclui-se que a técnica de psicoeducação é uma importante ferramenta no tratamento de usuários de álcool e/ou outras drogas como forma de Prevenção e Promoção de Saúde já que, através da mesma, os usuários tiveram uma diminuição dos quadros de ansiedade. Com isso, os conflitos foram reduzidos e, consequentemente, houve diminuição de recaídas com uso de álcool e/ou outras drogas ou posterior uso de medicação.

Palavras-chave: Saúde Mental. Psicoeducação. Prevenção e Promoção de Saúde. Terapia Cognitivo Comportamental. Centro de Atenção Psicossocial Álcool e outras Drogas.

\section{Abstract}

This paper reports the experience of an intervention project in Psychology, focused on Prevention and Health Promotion, carried out in Psychosocial Care Center - Alcohol and Other Drugs (CAPS AD) through psychoeducation workshops. For that, eight meetings were held, in workshop format, from August to September 2017, with a workload of two hours per week. Ten users of the service participated in the meetings, being the majority men in the age range of 36 to 57 years. The main purpose of the workshops was to teach relaxation techniques, through the exercise of diaphragmatic breathing, related to psychological techniques for subjects to reflect on themselves in the world. As a result, the participants reported a decrease in anxiety and tension producing, as an effect, changes in their daily habits, both in sleep,food and in relation to family conflicts, becoming more active in relation to their own treatment and taking off the focus of medical attention and the search for medicalization of the symptoms. It is concluded that the psychoeducation technique is an important tool in the treatment of users of alcohol 
and / or other drugs as a form of Prevention and Health Promotion since, through the same, users had a decrease in the anxiety. As a result, the conflicts were reduced and, consequently, there was a decrease in relapses with alcohol and/or other drugs or subsequent use of medication.

Keywords: Mental Health. Psychoeducation. Prevention and Health Promotion. Cognitive Behavioral Therapy. Psychosocial Care Center - Alcohol and Other Drugs.

\section{Introdução}

No Brasil a Luta Antimanicomial, datada do início da década de 70, produziu questionamentos e críticas sobre os modelos de tratamento para pessoas em sofrimento psíquico, modelos esses que isolavam tais pessoas do meio social, institucionalizando-as por longos períodos dentro de manicômios. A Luta Antimanicomial foi essencial para que ocorresse a Reforma Psiquiátrica no país, como a criação da Lei I0.2 I6 de abril de 200I (BRASIL, 200I), que levou a construção de serviços substitutivos ao modelo manicomial. Desde então, inúmeros estudos no campo de saber da Psicologia têm se dedicado para pensar a prática profissional na área das políticas públicas, tanto de Saúde Mental, quanto em outros setores dos Direitos Sociais, especialmente, no que se refere a relação da Psicologia com as políticas de Estado (LIMA, 2004; ANDRADE, 20II; SCARPARO; TORRES; ECKER, 20I4; ECKER; TORRES, 20I5a; 20I5b; PEDROSO; MEDEIROS, 2016; REIS et al., 20I7).

Os Centros de Atenção Psicossocial (CAPS) são parte da nova rede de políticas de Estado em Saúde Mental, criados para serem substitutivos às internações em hospitais psiquiátricos, na perspectiva da Prevenção e Promoção de Saúde, constituem-se como uma das principais estratégias do processo da Reforma Psiquiátrica. Nos CAPS são acolhidas pessoas com sofrimento psíquico com o objetivo de se estimular sua integração social e familiar, oferecer-lhes atendimento multidisciplinar e atividades que fortaleçam a responsabilidade e autonomia dos sujeitos no seu próprio tratamento (PEDROSO; MEDEIROS, 20I6).

Dentro dessa nova proposta de atendimento em Saúde mental foram estabelecidos três tipos de CAPS: o CAPS adulto, para atendimento de pessoas maiores de 18 anos com transtornos mentais; o CAPS Infantil, para atendimento de crianças e adolescentes até os 18 anos que possuem algum transtorno mental ou fazem uso problemático de substâncias psicoativas; e o CAPS AD, voltado para o atendimento de pessoas maiores de 18 anos que fazem uso problemático de substâncias psicoativas (WETZEL et al., 2008).

A palavra 'problemático' foi utilizada para se referir aquelas situações de uso que colocam tanto o sujeito que faz uso em risco, assim como, outras pessoas em sua volta. É reconhecido que existe em nossa sociedade uma diversidade de modalidades de uso das substâncias psicoativas, nas quais nem todas levam a uma situação problemática, ou seja, as drogas não são o problema em si, mas são as relações que os sujeitos estabelecem com as drogas que podem se tornar um problema (TORRES; ECKER, 20I7). Porém, o uso problemático de álcool e outras drogas tem se tornado um importante problema de saúde pública no Brasil e no mundo, gerando vários reflexos negativos na vida biopsicossocial de uma parcela da população (ANDRADE, 20I I).

É a partir da complexidade biopsicossocial da vida do usuário de álcool e/ou outras drogas que o CAPS $A D$ intervêm, buscando atuar num cuidado que priorize tratamentos humanizados, sem ser reclusório ou absenteísta, trabalhando conforme o modelo antimanicomial, na lógica da Redução de Danos'. Assim, um dos focos principais do tratamento no CAPS AD é de ser um espaço de Prevenção e Promoção de Saúde, atuando como processo de reconstrução dos laços dos usuários(as) com a comunidade, uma vez que na sua maioria encontram-se com os vínculos familiares, comunitários e de trabalho rompidos ou fragilizados devido ao longo tempo de uso e abuso de substâncias psicoativas. Para tanto, utiliza-se como uma das ferramentas de tratamento a Redução de Danos, entendendo esse modelo como uma forma de acolher a diversidade de usuários(as) com seus diferentes objetivos, ao invés de pensar a abstinência total como única forma de tratamento ou cuidado. Nesse sentido, a Redução de Danos possibilita uma melhor adesão dos usuários(as) no serviço e ao tratamento, já que considera a singularidade dos sujeitos na formulação do plano terapêutico.

\footnotetext{
I A Redução de Danos é uma prática que visa minimizar as consequências diversas criadas pelo uso de drogas tanto na saúde quanto na vida econômica e social dos usuários e seus familiares. A Redução de Danos está regulada pela Portaria $N^{\circ}$ I.028 de I ${ }^{a}$ de Julho de 2005 do Ministério da Saúde: http://bvsms.saude.gov.br/bvs/saudele gis/gm/2005/prt I028_01_07_2005.html
} 
Neste contexto, as atividades realizadas no CAPS AD baseiam-se nos princípios da universalidade, integralidade, equidade, intersetorialidade, humanização do atendimento e participação social preconizada pelas propostas do Sistema Único de Saúde (SUS) (BRASIL, 2006). A atuação da equipe multiprofissional no CAPS AD é pautada pelo diálogo, comunicação e interação entre a equipe, na troca dos saberes técnicos, em prol de oferecer atendimento qualificado e humanizado aos usuários (PEDUZZI, 1998). Dentre suas ações, os CAPS AD trabalham com a proposta de oficinas terapêuticas, que buscam a produção de saúde, reconhecimento e valorização das diferentes subjetividades criando, assim, novas formas de se relacionar, de ser e estar no mundo, modificando aqueles comportamentos que levam ao adoecimento.

No caso do uso problemático de drogas, as oficinas terapêuticas estimulam o processo de autocuidado e da autonomia do usuário(a) em lidar com as substancias psicoativas no cotidiano (LIMA, 2004). O conceito de autonomia, assim, não significa exclusão e individualização dos sujeitos no seu cuidado, mas, pelo contrário, busca ampliar e desenvolver no sujeito uma auto responsabilização, incluindo, também, uma maior quantidade de parceiros na sua rede de cuidados e tratamentos: "autonomia, no contexto da Reforma Psiquiátrica brasileira, é sempre construída na relação com o outro, em um exercício de compartilhamento de diferentes pontos de vista, numa experiência de cogestão" (CAMPOS et al., 2012, p.9).

Essa autonomia se torna possível quando os sujeitos conhecem seus limites e potencialidades, afirmando-se enquanto agentes das suas vidas, preventores e promotores de sua própria saúde. Sem o conhecimento sobre si no mundo, se torna mais difícil optar e ter autonomia para decidir por sua vida. Foi pensando na ampliação dos conhecimentos de si, com consequente desenvolvimento da autonomia dos sujeitos, que foi proposta atividade de psicoeducação em uma das oficinas terapêuticas no CAPS AD. Assim, este artigo relata de modo breve a experiência de um projeto de intervenção em Psicologia através de oficinas de psicoeducação, com o foco na Prevenção e Promoção de Saúde, realizado em um Centro de Atenção Psicossocial Álcool e outras Drogas (CAPS AD).

\section{Materiais e métodos}

O projeto de intervenção no CAPS AD foi realizado através de oficinas ${ }^{2}$ embasadas pela técnica terapêutica de psicoeducação. A psicoeducação é uma ferramenta de intervenção de diversas teorias da psicologia e, na presente proposta, utilizou-se a abordagem da Terapia Cognitivo Comportamental (TCC). Nela, se relaciona instrumentos psicológicos e pedagógicos para ensinar o sujeito que faz uso abusivo de álcool e outras drogas sobre sua patologia oferecendo, para tanto, práticas alternativas para lidar com os pensamentos e comportamentos que desencadeiam o uso de substâncias. Ela estimulando, também, a autonomia dos sujeitos nos cuidados com sua saúde física e psíquica (LEMES; NETO, 2017).

Assim, foram realizados oito encontros em formato de oficinas no período de agosto a setembro de 2017, com carga horária de duas horas semanais. Participaram desses encontros 10 usuários do serviço, sendo a maioria homens na faixa etária de 36 a 57 anos. As oficinas tiveram como principal dispositivo ensinar técnicas de relaxamento, através do exercício da respiração diafragmática, relacionadas a técnicas psicológicas para os sujeitos refletirem sobre si no mundo. A técnica de respiração diafragmática ajuda a ativar o sistema autônomo parassimpático de nosso cérebro, responsável por inibir a ação do sistema simpático e restaurar a sensação de relaxamento. Por isso ela é uma técnica tão importante para os casos de ansiedade. Além disso, é um bom exercício de distração, ajudando a pessoa a se desvencilhar da hipervigilância aos sintomas físicos e cognitivos da crise (NETO, 20I I).

O uso da psicoeducação em processos terapêuticos ensina o paciente sobre como essa técnica poderá ser útil no tratamento de sua ansiedade, tensão, medo, fissura, dentre outras sensações relacionada a vida. A técnica de psicoeducação inicia com a respiração diafragmática, que consiste em concentrar o ar na região do diafragma (músculo que fica na altura do estômago), fazendo a barriga estufar e voltar ao normal ao puxar e soltar $\mathrm{o}$ ar, respectivamente. Deve ser feita lentamente, geralmente, usando uma contagem até 3 lentos segundos para inspirar e até 6 para expirar. Para verificar se o procedimento está correto, pode-se colocar uma das mãos sobre o diafragma, para senti-lo subir e descer. Nesse processo, os sujeitos já começam a desenvolver consciência sobre os limites dos seus corpos, além disso, fazer a respiração lentamente é uma

${ }^{2}$ Por se tratar de um trabalho com o intuito exclusivamente de educação e ensino, de acordo com a Resolução n ${ }^{\circ} 510$, de 07 de abril de 2016, não fora registrada e nem avaliada pelo sistema CEP/CONEP. 
aprendizagem sobre o controle do corpo e as emoções, via autoconhecimento. Em seguida, com a mão direita se segura firme o pulso da mão esquerda por 5 minutos, conjuntamente, utilizando a respiração diafragmática lentamente. Após, solta-se a mão e cada pessoa avalia o resultado da prática dando uma nota de 0 até 10 , sendo o nível de tensão sendo 0 (zero), quando não houve alteração, e 10 quando o nível de tensão diminuiu totalmente.

O próximo exercício é esfregar as mãos por um período de 3 minutos e, paralelamente, centrar na respiração. Após, coloca-se as mãos no rosto, com os olhos fechados, fazendo uma automassagem no rosto, nuca e ombro. Finalizando, cada pessoa avalia como estava o nível de ansiedade e tensão inicial e relata como ficou após a participação no exercício. A proposta de conversar e dar notas a respeito dos níveis de tensão, que a pessoa sentiu ou está sentindo, ajuda ela a ir criando consciência e parâmetros cada vez mais aguçados para lidar melhor com seu corpo e emoções. Essa técnica é muito utilizada também no tratamento dos transtornos do sono, uma vez que tem o caráter de relaxamento. Ao praticar a respiração diafragmática, a pessoa passa a observar melhor a respiração, seu corpo no mundo presente, e a dar menos atenção aos demais pensamentos.

Além dessa atividade, foram realizadas dinâmicas interpessoais, visando refletir sobre si no mundo, e dinâmicas de movimento corporal, para soltar as tensões musculares e alongamento corporal. Outras técnicas psicológicas realizadas no grupo tiveram como finalidade a interação grupal, tais como: conversação, trocas de experiências, compartilhamento sobre informações da rede socioassistencial, relatos de vivências positivas, confecção de lanches para serem consumidos pelos membros do grupo, dentre outras atividades lúdicas terapêuticas. As oficinas de psicoeducação, além de ensinarem a técnica de respiração diafragmática, possibilitaram um espaço de conversa em que os usuários pudessem pensar sua vida para além do uso problemático de substâncias, criando estratégias de enfrentamento da ansiedade que desencadeia o uso, fortalecendo a comunicação e os comportamentos adaptativos para melhor inserção e interação social. No final de cada encontro, foi reservado um tempo para que cada usuário relatasse suas sensações com o uso da técnica de relaxamento na oficina, assim como, as possíveis experiências pessoais do uso da técnica realizadas fora do CAPS AD. Tais relatos foram anotados para uma análise mais detalhada das experiências.

\section{Resultados e Discussão}

Como resultado terapêutico da psicoeducação, através das técnicas de relaxamento e de autoconhecimento, os participantes relataram diminuição da ansiedade e tensão levando, assim, a mudanças em seus hábitos diários, tanto no sono, como na alimentação e em relação aos conflitos familiares. Com isso, passaram a ter uma atitude mais ativa no próprio tratamento e tirando o foco do atendimento médico e da busca pela medicalização dos sintomas. Alguns relatos apresentam elementos dessas mudanças: "Com o relaxamento passei a tomar menos medicamentos" (sic); "Faço o relaxamento do pulso quando estou em casa e não discuto mais com minha mãe"(sic); "minhas dores nas costas diminuíram, sinto-me melhor"(sic); "controlei a respiração e ajudou a me acalmar"(sic); "meu sono melhorou, ajudou-me a dormir mais e melhor, melhorou muito"(sic); "o relaxamento diminuiu muito a minha ansiedade, estou mais calmo, tranquilo"(sic); "Quando sinto falta do remédio intensifico o exercício da respiração e assim diminuo um pouco o uso de muito medicamento"(sic).

O que se verificou com as oficinas é que muitos usuários buscavam um tratamento como se nele fossem encontrar uma cura repentina aos seus problemas, crendo, inclusive, que a medicação era o único caminho para essa cura. Exemplo dessa situação foi relatado por um usuário em um dos encontros, disse ele: "tratamento sem medicação não é tratamento" (sic). Assim, o espaço a eles proporcionado pelas oficinas possibilitou a discussão e o esclarecimento de modo que pudessem compreender que a medicação é apenas um dos componentes de um tratamento que envolve muitas variáveis. Dentre as variáveis de um tratamento está o fato de que muitos usuários não precisam de medicação para modificar sua relação com o uso de substância. Discussões evidenciam que a medicalização tem transformado culturalmente as populações, declinando sua capacidade de enfrentamento autônomo da maior parte dos adoecimentos e das dores cotidianas. Isso desemboca no consumo abusivo e contra produtivo de medicamentos, gerando dependência excessiva dos mesmos e alienação de si sobre seu próprio corpo e saúde (TESSER, 2006).

Outra problemática discutida nos encontros foi o uso correto da medicação pois, mesmo a maioria dos usuários acreditando ser o remédio a única via de cura, muitos faziam uso incorreto do mesmo. Verificava-se 
que tomavam a medicação sem seguir a prescrição ou interrompiam quando acreditavam que já estavam curados. Ainda, alguns faziam uso de substâncias que interferiam na eficácia da medicação, comprometendo o tratamento medicamentoso. Pela psicoeducação, aprendendo sobre os medicamentos e seus efeitos no corpo e comportamento, incluindo discutir sobre a interferência das interações com outras substâncias e a importância do tempo de tratamento de cada componente químico, contribuiu-se com o desenvolvimento de uma consciência sobre o uso correto dos medicamentos, tornando os usuários protagonistas e autônomos na própria condução de sua terapêutica.

Para alguns usuários foi possível, inclusive, fazer a diminuição do uso de medicamentos após a articulação entre as oficinas e a avaliação psiquiatra do CAPS AD. Vemos isso nos seguintes relatos "Eu tomava seis tipos de medicamentos, para tudo. Agora o psiquiatra diminuiu dois. Agora tomo quatro, e me sinto bem depois que comecei a frequentar essa oficina e a fazer o relaxamento" (sic). Já outro usuário diminuiu o uso da medicação pela metade: "Eu estava tomando seis comprimidos durante 8 meses. Agora o clinico geral avaliou e passei a tomar menos, ele disse que estou melhorando. Agora tomo só três" (sic).

Nessa breve reflexão sobre a experiência de oficinas terapêuticas em um CAPS AD, conclui-se que a técnica de psicoeducação é uma importante ferramenta da Psicologia no tratamento de usuários de álcool e/ ou outras drogas como forma de Prevenção e Promoção de Saúde já que, através dela, os usuários tiveram uma diminuição dos quadros de ansiedade. Com isso, os conflitos foram reduzidos e, consequentemente, houve diminuição de recaídas com uso de álcool e/ou outras drogas ou posterior uso de medicação.

Concomitantemente, os encontros também propiciaram uma maior interação entre os participantes do grupo, através de conversação, confecção de lanches, atividades lúdicas, de relaxamento, trocas de experiências e vivências positivas, estimulando as diversas maneiras de expressão e integração das singularidades de cada um. Com isso, na lógica da Prevenção e Promoção de Saúde, produziu-se um cuidado próximo dos princípios da Reforma Psiquiátrica, da Redução de Danos e das diretrizes do Sistema Único de Saúde, no que se refere a um cuidado humanizado e integral, que leve em consideração aspectos biopsicossociais na formulação dos projetos terapêuticos.

\section{Considerações finais}

Através da experiência proporcionada pelas oficinas, conclui-se que a técnica de psicoeducação é uma importante ferramenta terapêutica da Psicologia no tratamento de usuários de álcool e outras drogas. Como estratégia de Prevenção e Promoção de Saúde, os usuários obtiveram uma diminuição aos quadros de ansiedade que desencadeavam os conflitos, o uso de substâncias e o posterior abuso da medicação. O foco na Prevenção e Promoção de Saúde possibilitou uma vivência que reflete o conceito ampliado de saúde, isto é, saúde para além da 'cura das enfermidades', em que o sujeito precisa ser analisado e cuidado de modo integral, dentro de sua complexidade social e pessoal, como orientam os princípios do SUS.

Sendo assim, a psicoeducação é parte do cuidado em saúde, pois através dela o sujeito se apropriará de ferramentas e recursos que possibilitarão uma melhor adaptação à seu meio, com consciência e crítica, podendo, assim, ter outras opções de escolhas e modos de viver salutares. Desenvolver espaços de psicoeducação dentro das instituições de cuidado, como o CAPS AD, possibilita que os profissionais do local ampliem suas ações, tornando as políticas públicas do Estado um espaço para além da 'cura' de doenças, mas, principalmente, ferramenta preventora e promotora de saúde. Previnir e promover saúde nas políticas públicas possibilita superar ações com viés de 'embotamento das subjetividades' ou medicalização excessiva dos sintomas (COSTA-ROSA, 20I I), transformando as instituições em parceiras ativas para a Prevenção e Promoção de Saúde de forma mais complexa e, portanto, eficaz.

Referente a utilização dos medicamentos psicotrópicos na população brasileira, segundo dados obtidos junto à Agência Nacional de Vigilância Sanitária (ANVISA, 2007), dentre os psicofármacos mais consumidos no Brasil encontram-se os ansiolíticos, antidepressivos e emagrecedores. Com isso, a ANVISA evidenciou que, dentre os princípios ativos com maior consumo no país, no período de 2007 a 2010, encontram-se o clonazepam (Rivotril), bromazepan (Lexotan) e alprazolan (Frontal). No ano de 2010, segundo informações obtidas junto às farmácias cadastradas, a ANVISA constatou que foram dispensadas 10,5 milhões de caixas de clonazepan, o que indica uma quantidade elevada do uso deste medicamento. Nesse sentido, a prescrição de psicofármacos se mantêm excessiva: 
[...] da forma como vem sendo praticada, poderá se constituir numa nova maneira de controle através da drogadição da população social que, refém de modalidades terapêuticas psicofarmacológicas, pode sacrificar seriamente qualquer perspectiva de autonomia e liberdade (FERRAZZA; ROCHA; LUZIO, 20I7, p. I3).

Constata-se que, atualmente, o governo tem gasto bilhões ${ }^{3}$ com o fornecimento de medicações para a população, sendo que grande parte dessas medicações podem estar sendo usadas de forma incorreta, levando a um prejuízo na saúde do usuário e, também, um desperdício do dinheiro público. O modo como se tem receitado excessivamente a medicação, em detrimento de outras práticas humanizadas, eficazes e salutares, pode estar dificultando a produção de autonomia e responsabilização do usuário. Em muitos casos, eles têm ficado dependentes do remédio, sem desenvovler outras estratégias de auto cuidado, ou têm apresentado problemas físicos e/ou psicológicos decorrentes do uso incorreto ou devido aos efeitos colaterais das substâncias psicotrópicas. Tais gastos públicos e prejuízos na saúde poderiam ser reduzidos com ações de Prevenção e Promoção de Saúde, através da psicoeducação, como mostrou o resultado das oficinas desenvolvidas no CAPS AD.

A proposta da psicoeducação, como uma das ferramentas terapêuticas com usuários de álcool e outras drogas, seja pela técnica da respiração diafragmática ou técnicas psicológicas de autoconhecimento e interação social, ajudaram usuários a diminuir pela metade o uso de medicamentos. Seria, então, as ações de Promoção e Prevenção de Saúde um recurso importante para redução da excessiva medicalização de sintomas? A partir dos resultados positivos da presente experiência, sugere-se que sim. Com isso, apontamos a importância da realização de outras experiências e pesquisas em psicoeducação, nas diferentes políticas públicas brasileiras, para que se possam buscar indicadores que avaliem e quantifiquem o impacto dessa ferramenta terapêutica da Psicologia no cuidado da população.

\section{Referências}

ANDRADE, T. M. de. Reflexões sobre políticas de drogas no Brasil. Ciênc. saúde coletiva, v. I6, n. I2, pp.4665-4674, 20I I. Disponível em: http://www.scielo.br/pdf/csc/vl6n I 2/I5.pdf Acesso em: 03 jan. 2019.

ANVISA. Agência de Vigilância Sanitária. Boas Práticas de Medicamentos de Preparações Magistrais e Oficinas para uso Humano em Farmácias. RDC N. 67, de 08 de outubro de 2007. D.O.U. Brasília, DF, 2007.

BRASIL. Lei n 10.2 I6, Lei da Reforma Psiquiátrica

de 06 de abril de 200 I. Diário Oficial da União, 200 I.

Ministério da Saúde. Uma análise da situação de saúde no Brasil.. Brasília: Ministério da Saúde, Secretaria de Vigilância em Saúde, Departamento de Vigilância Análise de Situação em Saúde, 2006.

. Lei n $^{\circ}$ 8.080, de 19 de setembro de 1990. Sistema Único de Saúde (SUS), 1990.

CAMPOS, et al. Guia da Gestão Autônima da Medicação - GAM - DSC/FCM/UNICAMP; AFLORE IPUB/UFRJ; DP/ UFF; DPP/UFRGS, 2012. Disponível em: http://www.redehumanizasus.net/sites/default/files/guia_da_gestao_autonoma_da_medicacao.pdf. Acesso em 03 jan. 2019.

COSTA-ROSA, A. Operadores fundamentais da atenção psicossocial: contribuição a uma clínica dos processos de subjetivação na saúde coletiva. Tese de livre-docência não publicada. Universidade Estadual Paulista "Julio de Mesquita Filho", Assis, SP, 20II.

ECKER, D. D.; TORRES, S. Relato de experiência no programa PIM-PIA: políticas públicas e primeira infância. Percurso Acadêmico, Belo Horizonte, v. 5, n. 10, jul./dez. 2015a. Disponível em: http://seer.pucminas.br/index.php/perc ursoacademico/article/view/8420 Acesso em: 10 jan. 2019.

ECKER, D. D.; TORRES, S. Política de cotas étnicas no ensino superior: análise de narrativas de sujeitos do meio universitário. Emancipação, Ponta Grossa, v. 15, n. I, 20 I5b. Disponível em: http://seer.pucminas.br/index.php/ percursoacademico/article/view/8420 Acesso em: 10 jan. 2019.

FERRAZZA, D. A.; ROCHA, L. C. da; LUZIO, C. A. Medicalização em um serviço público de Saúde Mental: um estudo sobre a prescrição de psicofármacos. Revista Interinstitucional de Psicologia, 6 (2), jul - dez, 20I 3, 255-265. Disponível em: http://pepsic.bvsalud.org/pdf/gerais/v6n2/v6n2a08.pdf Acesso em: 03 jan. 2019.

${ }^{3}$ Em 2015 foram gastos mais de 14 bilhões com medicação (Fonte: Grupo Técnico Interinstitucional de Discussão do Financiamento do SUS). 
LEMES, C.B., NETO, J.O. Aplicações da Psicoeducação no Contexto da Saúde. Temas de Psicologia, v.25, n. I p.I728. DOI: 10.978 8/TP2017.I-02. 2017

LIMA, E. A. Oficinas terapêuticas em Saúde mental: sujeito, produção cidadania. Rio de janeiro, p.59-8I, 2004.

NETO, A. R. N. Técnicas de respiração para a redução do estresse em terapia cognitivo-comportamental. Arquivos Médicos do Hospital e das Faculdades de Ciências Médicas da Santa Casa São Paulo, 201 I, 56 (3), p: I58-68. Disponível em: http://www.fcmscsp.edu.br/files/AR09.pd f. Acesso em: 03 jan. 2019.

PEDROSO, R., MEDEIROS, R.H. A. de. Efeitos da reforma psiquiátrica no processo de acolhimento no CAPS AD em Viamão, RS. Journal Health NPEPS, I (2), 23I-245, 20l6. Disponível em: http://periodicos.unemat.br/inde x.php/ jhnpeps/article/view/I589/15I5. Acesso em: 04 jan. 2019.

PEDUZZI, M. Equipe multiprofissional de saúde: conceito e tipologia. Revista de Saúde Pública, 35 (I), p. I03-I09, Campinas, 1998.

RANGÉ, B.; MARLATT, G. A. Terapia cognitivo-comportamental de transtornos de abuso de álcool e drogas. Revista Brasileira de Psiquiatria, p.88-95, 2008. Disponível em: http://www.scielo.br/pdf/rbp/v30s2/a06v 30s2.pdf Acesso em: 04 jan. 2019.

REIS, C. et al. Como criar o caos social: mídia e políticas sobre drogas. Percurso Acadêmico, Belo Horizonte, v. 7, n. I4, jul./dez., 20I7. Disponível em: http://ibict.pucmin as.br/index.php/percurso academico/article/view/I58I6/I3365. Acesso em: 10 jan. 2019.

SCARPARO, H. B. K; TORRES, S; ECKER, D. D. Psicologia e ditadura civil-militar: reflexões sobre práticas psicológicas frente às violências de estado. Rev. Epos, Rio de Janeiro, v.5, n. I, p.57-78, jun. 2014. Disponível em: http://pepsic.bvsalud.org/scielo.php?script=sci_arttext\&pid=S2 I78-700X20 $4000100004 \&$ Ing =pt\&nrm=iso. Acesso em: 10 jan. 2019.

TESSE, C. D. Medicalização social (I): o excessivo sucesso do epistemicídio moderno na saúde. Interface , Botucatu , v. 10, n. 19, p. 6I-76, June, 2006. Disponível em: http://www.scielo.br/scielo.php?script=sci_art text\&pid=S|4|4-32832006000 I00005\&lng=en\&nrm=iso. Acesso em: 03 jan. 2019.

TORRES, S., ECKER D.D. Capacitação de profissionais sob a perspectiva da redução de danos: drogas, vamos pensar! Rev. Elet. Cient. UERGS, v. 3, n. I, p.39-62. Disponível em: http://revista.uergs.edu.br/index.php/revuergs/arti cle/ view/494/144. Acesso em: 03 jan. 2019.

WETZEL, C.; KANTORSKI, L. P.; SOUZA, J. de. Centro de Atenção Psicossocial: trajetória, organização e funcionamento. Revista Enfermagem Uerj, Rio de Janeiro, v. I6, n. I (jan./mar. 2008), p. 39-45, 2008. 